\title{
Permporometry. The determination of the size distribution of active pores in UF membranes
}

\author{
F.P. Cuperus, D. Bargeman and C.A. Smolders \\ University of Twente, Dept Chemical Technology, P O Box 217, NL-7500 AE Enschede (Netherlands)
}

(Received April 23, 1991, accepted in revised form March 2, 1992)

\begin{abstract}
Permporometry is a method by which the characteristics of the interconnecting 'active' pores of an ultrafiltration membrane can be measured It 2 s these 'active' pores that are responsible for the actual membrane performance Application of permporometry on different membrane types, including ceramic as well as polymeric membranes, shows that the method can provide objective information on the 'active' pore sizes present
\end{abstract}

Keywords permporometry, ultrafiltration, actıve pore sıze, pore size distribution

\section{Introduction}

For the measurement of pore sizes present in mesoporous media a variety of techniques are avallable. The majority of these originate from ceramic materials science, where it is of particular interest to know the pore size distribution and the pore volume, irrespective whether it concerns dead-end or interconnecting pores. The characterization of UF membranes, however, is aimed at the determination of characteristic membrane properties in order to predict the transport properties and membrane performance as much as possible. To this end, it is crucial to measure the sizes of those pores which really contribute to the permeability In the case of UF membranes these so-called 'ac-

Correspondence to Dr Ir F P Cuperus, ATO Agrotechnologie, Agricultural Research Institute, P O Box 17, NL6700 AA Wageningen (Netherlands) tıve pores' are present in the 'active' skın layer of the membrane.

Most of the routine characterization methods cannot discriminate between the 'active' pores in the skin and 'inactive' pores present in the skin or in the supporting layer. Exceptions are the gas permeability method $[1,2]$ and the liquid-liquid displacement technique [3,4]. These methods have other disadvantages and are not always capable of generating accurate, independent, characteristic data The significance of the gas permeability method is limited, because only mean pore sizes are measured and, as Altena et al have shown [2], the quantitative values of these pore sizes are highly ambiguous With the second method mentioned above (the liquid-liquid displacement technique), the pore size distribution of a membrane can be measured, but the results are influenced by the experimental conditions [3]. These ambiguties show the need for other 
characterızation methods by which the sizes of active pores can be determined.

Permporometry, introduced by Eyraud et al. $[5,6]$ and modified by Katz et al. [7,8], is a relatively new characterization method to evaluate the active pores of an UF membrane. It is based on the controlled stepwise blocking of pores by condensation of a vapour, present as a component of a gas mixture, and the simultaneous measurement of the gas flux through the membrane. In this paper we report on permporometry measurements using an experimental apparatus and procedure which provides a very well-defined (and easy to model) transport regime and a fast adjustment of equilibrium conditions.

\section{Theoretical}

\section{Capillary condensation}

The nature of physisorption processes of condensable vapour is usually divided in different stages [9]. In the beginning of the process, at low relative pressures, only adsorption of vapour molecules to the pore wall is assumed to occur This adsorption is restricted to the socalled ' $t$-layer' with a maximum thickness in the order of a few molecules. At higher values of the relative pressure the adsorption process is followed by capillary condensation, i.e. the condensation of a vapour, commencing in the smallest pores. As the pressure is progressively Increased, wider pores are filled until at the saturation pressure the entire system is filled with condensate. The relative pressure at which pore filling starts depends on the radius of the capllary and can be calculated from the Kelvin relation (1):

$\ln p_{\mathrm{r}}=\left(\frac{-\gamma v}{R T}\right) \cos \theta\left(\frac{1}{r_{\mathrm{k} 1}}+\frac{1}{r_{\mathrm{k} 2}}\right)$

In the application of the Kelvin reaction it is generally accepted to assume that $\theta=0$. This simplifies eqn. (1) and permits the direct calculation of a pore radius corresponding to a certain relatıve pressure [9].

During desorption a behaviour similar to adsorption is shown. Now a liquid containing pore will not empty before the vapour pressure falls below the equilibrium pressure given by eqn (1). The desorption process is pictured schematically in Fig. 1. The adsorption and desorption processes are not necessarily defined by the same curvature of the liquid-gas interface which often leads to a hysteresis phenomenon. For instance, during adsorption in a cylindrical pore the meniscus has a cylındrical shape [in eqn. (1): $r_{\mathrm{k} 1}=r_{\mathrm{k}}$ and $r_{\mathrm{k} 2}=\infty$ ] whereas during desorption the liquid-gas interface is hemispherical [in eqn. (1). $r_{\mathrm{k} 1}=r_{\mathrm{k} 2}=r_{\mathrm{k}}$ ], and eqn (1), with $\theta=0$, turns into:

$\ln p_{\mathrm{r}}=\left(-2 \gamma v / R T r_{\mathrm{k}}\right)$

The Kelvin radius $r_{k}$ found from eqn. (1A) is the radius of curvature of the liquid-gas interface, which is equal to the pore radius minus the 't-layer' thickness of the adsorbed film on the pore wall (Fig. 1). The relationship between the true pore radius $r_{\mathrm{p}}$ and the Kelvin radius $r_{\mathrm{k}}$ thus is:

$r_{\mathrm{k}}=r_{\mathrm{p}}+t$

in which $t$ is the thickness of the adsorbed $t$ layer.

The thickness of the t-layer depends on the relative pressure. In classical adsorption studies this thickness is calculated from separate adsorption experiments which are performed using homogeneous non-porous reference surfaces, preferably made of the same material as the porous medium This approach is very laborious and therefore an approximation, explained furtheron on this paper, was used to calculate the t-layer thickness directly from permporometry data

Capillary condensation provides the possibility to block pores of a certain size with liquid, just by setting the relative pressure. In perm- 


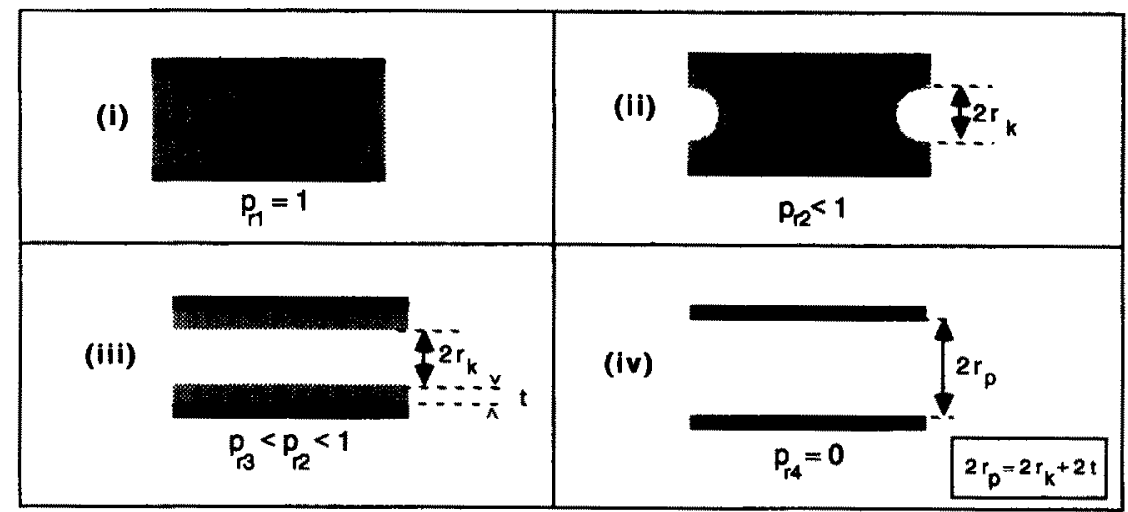

Fig. 1. Steps in the desorption process: (i) liquid filled pore, at saturation pressure, (ii) just before desorption starts, pore is still filled, (iii) just after evaporation is complete, t-layer remains, (iv) after complete desorption.

porometry this principle is combined with the measurement of the free diffusive transport through the open pores. Starting from a relative pressure equal to 1 , all pores of the UF membrane are filled so that unhindered gas transport is not possible. When the pressure is reduced, pores with a size corresponding to the vapour pressure applied, are emptied and become available for gas transport. By measuring the gas transport through the membrane upon decreasing the relative pressure, the distribution of the sizes of the active pores can be found. Of course, similar measurements can be done during the adsorption process, but the equilibrium of the adsorption process is more difficult to reach and therefore quantitative analysis of the desorption process is preferred.

\section{Counterdiffusion}

In this approach, the principle of counterdiffusion of two different gases, oxygen and nitrogen, in the absence of an overall 'mechanical' pressure gradient is used to monitor the transport through the membrane. Since in our case small pores $(<25 \mathrm{~nm})$ are measured, whereas the driving force for the transport is a concentration gradient of the two gases, a well-defined diffusion regime can be assumed [10]. At an overall pressure of about $100 \mathrm{kPa}$, the main transport mechanism is assumed to be Knudsen diffusion, which for a capillary structure is described by eqn. (3):

$J_{\mathrm{k}}=\left(\pi n r^{2} D_{\mathrm{k}} \Delta p\right) /(R T \tau l)$

in which the Knudsen diffusion coefficient $D_{\mathrm{k}}$ is:

$D_{\mathrm{k}}=0.66 r[(8 R T) /(\pi M)]^{0.5}$

\section{Experimental}

The experimental set-up (Fig. 2) is derived from those of Eyraud et al. [5,6] and Katz et

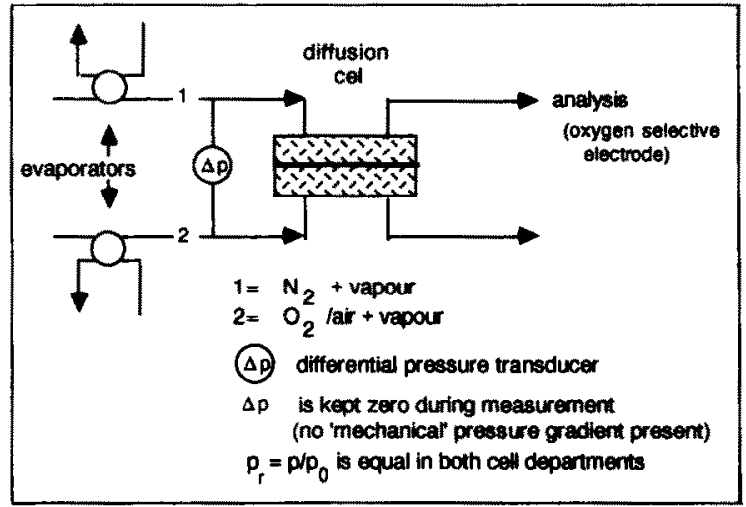

Fig. 2. Permporometry: experimental set-up. 
al $[7,8]$ and adapted to the conditions needed for counterdiffusion measurements. Along both sides of the membrane, a mixture of a condensable gas and a non-condensable gas is flushed The condensable gas can be any vapour, provided it has a reasonable vapour pressure and is inert with respect to the membranes to be characterized. The relative pressure of the vapour is maintained the same throughout the diffusion cell. The primary advantage of the apparatus presented here is the fact that the equilibrium between gas mixture and capillary condensate is reached faster than with the apparatus described by Eyraud et al. [5] and Katz and Baruch [8]

The measurements start at a relative pressure equal to one, which means a minimum in diffusive transport. While the membrane is equilıbrated at progressively lower relative vapour pressures, the diffusive transport of oxygen through the membrane is measured with an oxygen selective electrode. After reaching a relative pressure of 0 , the process is reversed and the adsorption branch is measured. Equilibration times varied from 15 to $30 \mathrm{~min}$, depending on the amount of condensate that had to be adsorbed or removed. Using eqns. (1)-(4), the pore size distributions were calculated from the desorption branch.

Several membranes were characterized by the method described above. Ceramic membranes were supplied by the group of Burggraaf [11], Nuclepore $0015 \mu \mathrm{m}$ membranes (lot no. 86A9B14) were purchased from the Nuclepore Corporation and DDS GR61PP, manufactured by DDS Danmark, were supplied by the Dutch Institute for Dairy Research (NIZO)

A more detailed study was made of anisotropic polymeric membranes, synthesized from poly (2,6-dimethyl-1,4-phenylene oxıde) (PPO) and polysulfone (PSf) The PPO membranes were prepared from a $10 \mathrm{wt} . \%$ polymer solution in a mixture of trichloroethylene and octanol-1 in a weight ratio $78 / 22$. The PSf membranes were made from a $15 \mathrm{wt} . \%$ PSf solution in DMF In both cases the solutions were cast at room temperature to a thickness of 0.20 $\mathrm{mm}$ on a glass plate. The PPO films were coagulated in a methanol bath and the PSf films in a water bath. All solvents used were of analytıcal grade.

In principle, the condensable gas should not alter the porous matrix and the vapours must be as inert as possible. To investigate the effect of different condensable gases, four different adsorbates, i e. ethanol, methanol, tetrachloromethylene and cyclohexane (p.a quality, Merck) were used.

\section{Results and discussion}

\section{Ceramıc $\gamma$-alumina membranes}

In Fig. 3 the oxygen flux through an alumina membrane during the adsorption and desorption of cyclohexane vapour is shown. This typ1cal flux-relative pressure plot can be divided into three main areas. In the interval $1 \geqslant p_{\mathrm{r}}>0.55$ all the pores are blocked with condensate and free gas diffusion is impeded At relative pressures between 0.55 and 0.3 , the flux increases with decreasing relative pressure be-

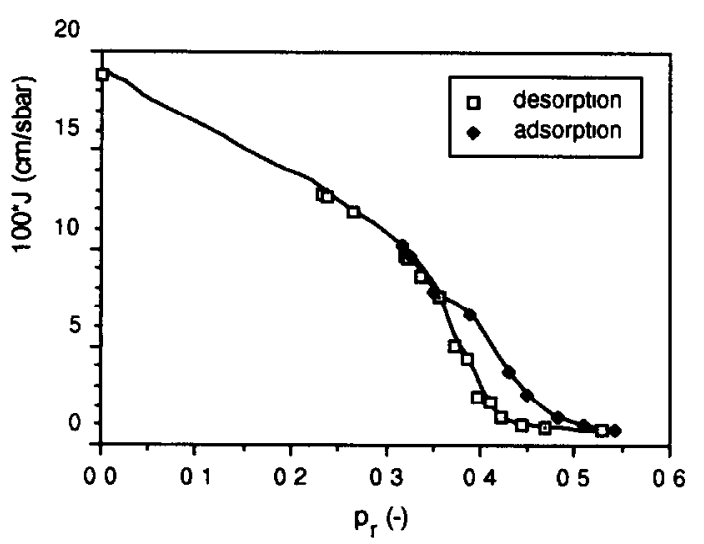

Fig 3 Permporometry adsorption and desorption curve for a $\gamma$-alumina membrane with cyclohexane used as adsorbate 
cause of an increasing number of open pores. In this interval the capilary condensation process takes place. The flux-pore size plot, given in Fig. 4. was calculated from the desorption data and assuming a capillary model (eqn. 1A) Frcm the measured diffusive flux values and the calculated Kelvin pore sizes, a number of pores was calculated using eqn (3).

In order to find the real pore size the Kelvin radı should be corrected for the adsorbed $t$ layer. Figure 3 shows that upon lowering the relative pressure beneath 0.3 , the flux through the membrane increases slightly It can be argueé that in thus region all pores with raôn $r_{k}$ are already empty and avalable for transport, but stinl the size of the pores increases a littie as the result of the desorption of the t-layer. When a uniform t-layer (with a thickness $t$ ) in all pores is assumed at $p_{\mathrm{r}}=03$, the effective pore size will increase from $r_{\mathrm{k}}$ to $r_{\mathrm{p}}=r_{\mathrm{k}}+t$ (eqn 2) upon decreasing the relative pressure from 0.3 to 0 So, by using the calculated pore size distribution (Kelvin radii) and the experimental values of the oxygen flux at relative pressures 03 and 0 , an estimation of the t-layer thickness can be made From the data found for cyclohexane on alumina a mean t-layer thickness of $05 \mathrm{~nm}$ (at $p_{\mathrm{r}}=0.3$ ) was calculated.

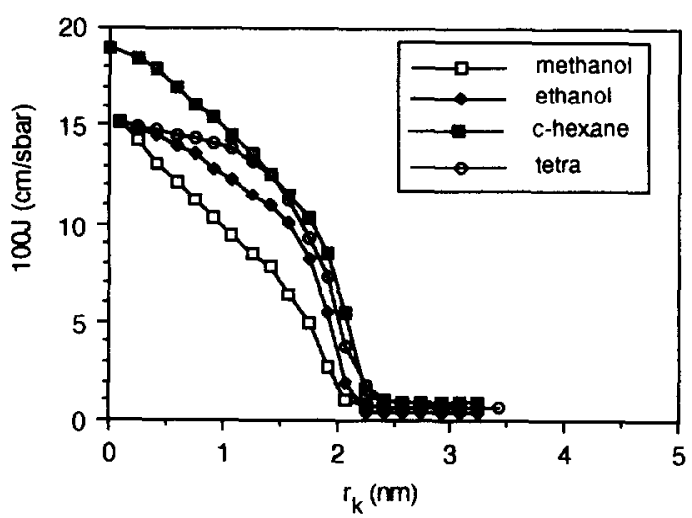

Fig \& Oxygen 7rax as a function of the hílvin rahus measured with different adsorbates found for $\gamma$-alumina membranes
In principle also the adsorption curve can be used for the pore size evaluation. However, the combination of the adsorption data with a capillary model gave substantially lower pore sizes than the desorption data. This may indicate that the pore shape of the alumua membrane is not cylindrical. Leenaars [12] concluded from classical adsorption-desorption studies that the alumina membranes contain slitshaped pores. If the ratio of the length to the width of these slits is not too high, this slitshaped pore model indeed is a better approximation.

To mvestigare the uñuence of bifferent aǹsorbates on the quantitative analysis, measurements using ethanol, methanoi and caroon tetrachloride as the condensable gas were carried out. Carbon tetrachloride and cyclohexane are considered as so-called Van der Waals gases which implies that their gas molecules are assumed to act as non-interacting hard spheres (similar to an ideal gas) with a finite volume Their physical behaviour is described by the Van der Waals equation. Methanol and ethanol are considered to be less ideal which may influence the permporometry measurements [13]

In Fig 4 the resuiting $J-r_{k}$ plots for the different vapours used, are shown The nominal Kelvin radı found with ethanol, tetrachloride and cyclohexane, differ only slightly When methanol is used, lower values of the Kelvin radil are found. This shift can be attributed to the difference in t-layer thickness. For ethanol, cyclohexane and carbon tetrachloride the calculated t-layer thicknesses (Table 1) are about the same $(\sim 0.4 \mathrm{~nm})$. This value corresponds to about one molecular layer, which is in good agreement with literature data on physical adsorption of organic molecules [9,14] In case of methanol the t-layer is significantly thicker: 07 $\mathrm{nm}$, which is an unexpectedly high value especially when compared with ethanol. In physical adsorption only Vàn dèr Waal's tôrces account for the interaction, which means that when the 


\section{TABLE 1}

Pore sizes $\left(r_{\mathrm{p}}\right)$ and t-layer thicknesses $(t)$ found for $\gamma$-alumina membranes and using different adsorbates

\begin{tabular}{lll}
\hline Adsorbate & $r_{\mathrm{p}}(\mathrm{nm})^{\mathrm{a}}$ & $t(\mathrm{~nm})$ \\
\hline Cyclohexane & 23 & 05 \\
Ethanol & 22 & 04 \\
Methanol & 23 & 07 \\
Carbon tetrachloride & 22 & 04 \\
\hline
\end{tabular}

${ }^{a}$ Nominal pore size

size of the molecules of the different adsorbates is comparable, the thickness of $t$-layers of these adsorbates is expected to be same. It follows therefore that the interaction between the alumina pore wall and the methanol molecules is substantially stronger than it is for the other condensable gases. This interaction has also been shown in other studies in which $\gamma$-alumina is used as catalyst in the oxidation of methanol [15].

The pore size distributions of the alumina membranes, corrected for the t-layer thickness agree very well with each other (Fig. 5). This indicates that, at least for such porous system, the pore size analysis is not dependent on the nature of the vapour used. It is clear that the $\gamma$ alumina membranes used, exhibit a very welldefined structure which is in agreement with results found with other characterization technıques. For instance, a very sharp pore size distribution $(r \sim 2 \mathrm{~nm})$ was also found with the gas adsorption-desorption technique and thermoporometry (Fig. 6) [12,13]. Also, the thickness of the active layer is known accurately (5 $\mu \mathrm{m})[11,12]$, which all together, should make the membrane a well-suited system to be used in a model study.

Usıng the experimentally determined Kelvin radii and the number of pores, a surface porosity and a volume porosity can be calculated. When the capillary model is applied with a tortuosity factor, $\tau=1$, the data result in a surface and volume porosity of $0.9-1.2 \%$ (Fig. 5 ) The
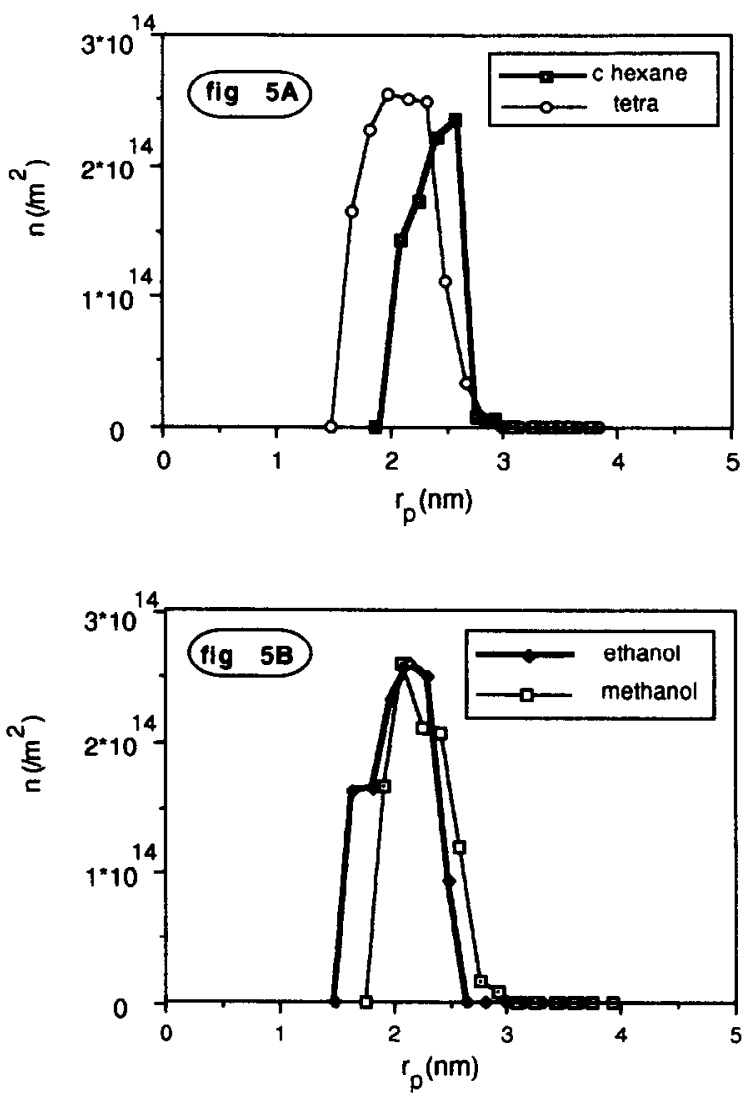

Fig 5 Pore size distributions of active pores of alumina membranes measured with different adsorbents in permporometry $(\tau=1)$ When corrections for tortuosity and resistance of the supporting layer are made, the number of pores $(n)$ is increasing by a factor of about 40 (see text)

other techniques already mentioned indicate a much higher value for the volume porosity $(\sim 45 \%)[11,12]$. One of the reasons for this large deviation is the extremely high tortuosity factor of the alumina membranes For many porous media the tortuosity factor has a value of $2-3$, but for the alumina system a value of 13 was found [12]. Another point is that, due to the very thick sublayer of the alumina membranes, the effective driving force across the top layer is only $30 \%$ of the total [11]. This means that, when the corrections for the tortuosity (so $\tau=13$ instead of 1 ) and the resistance of the 


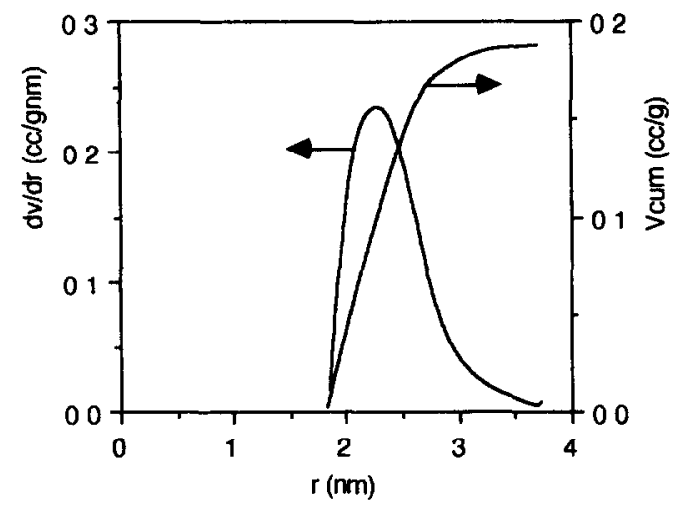

Fig 6 Pore size distributions for $\gamma$-alumina membranes using thermoporometry

sublayer are used, the volume porosity is calculated to be $35-50 \%$.

\section{Nuclepore membranes}

Nuclepore membranes are claimed to have a very well-defined capillary structure with a uniform pore size and thus a tortuosity equal to one $(\tau=1)$ can be assumed. Consequently these membranes should be very suitable for model studies. In this work membranes with a claimed pore radius of $75 \mathrm{~nm}$ were used.

The resulting pore size values found by permporometry (Fig 7) are indeed in reasonable agreement with the expected values. The experimental flux, however, appears to be 7 tımes higher than the value calculated from the data given by Nuclepore (number of pores: $6 \times 10^{12} / \mathrm{m}^{2}$, tortuosity: 1 , pore radius $7.5 \mathrm{~nm}$ )

Using electron microscopy (EM), the number of pores of similar membranes have been ınvestigated by a number of researchers $[16,17]$. Their results appeared to correlate very well with the Nuclepore values (also determined by EM) The only possibility to explain the apparent discrepancy is the tortuosity factor, which is suggested to be too high. A tortuosity factor lower than 1 can be related to a widening of the pores inside the membrane,

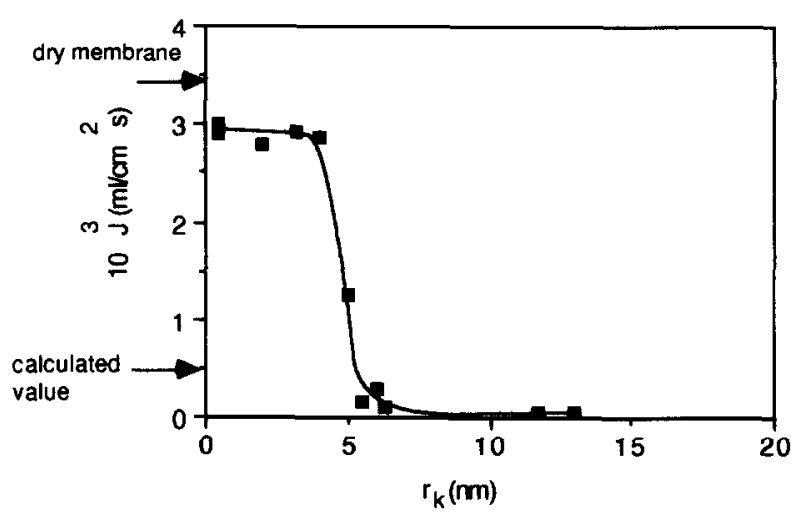

Fig 7 Oxygen flux as a function of the Kelvin radius measured with different adsorbates found for a Nuclepore 0015 $\mu \mathrm{m}$ membrane (calculated flux value using data given by Nuclepore $r=75 \mathrm{~nm}, n=6 \times 10^{12} / \mathrm{m}^{2}$, tortuosity $=1$ )

which accounts for a lower resistance for transport. From the experimental fluxes, the effective pore size (for water and gas transport') is estımated to be twice the size of the pore opening. Altogether this means that pores of a Nuclepore membrane have a narrow pore mouth with a size claimed by the manufacturer and a larger effective pore size inside the membrane which accounts for the main transport resistance

Since the pores of the Nuclepore membrane are relatively large, the thickness of the t-layer is of minor importance (Fig. 7) A remarkable effect, however, is that the t-layer thickness appears to be unchanged at very low relative pressures, which might be due to specific interaction (instead of only Van der Waals interactions). Going from a relative pressure of $\sim 0.7$ to 02 , the flux through the membrane is constant but when the relative pressure is decreased to 0 , a $10 \%$ increase in flux is found. This increase corresponds with a t-layer thickness of about $05 \mathrm{~nm}$ which is the thickness of about one monolayer of ethanol molecules [14].

\section{PPO membranes}

Pore size distributions of PPO membranes were determined using methanol and ethanol 
as the condensable component In Fig. 8 results for different samples of PPO membranes are given. It appears that the largest interconnecting pores present in PPO membranes have a size of about $15 \mathrm{~nm}$, although a large number of small pores are present too. Despite their low number, the larger pores mainly determine the performance of the membrane. Consequently the thickness of the adsorbed t-layer is not of significance; it is sufficient to know that it is very small. Using the same approach as before, the thickness for methanol was found to be 0.25 $\mathrm{nm}$. For ethanol the thickness was estimated to be $05 \mathrm{~nm}$.

In order to calculate the number of pores present in the membrane skin, all characteristic parameters in eqn (3) are needed. With the gold sol method [18] the skin thickness has been determined to be $0.2 \mu \mathrm{m}$ and (assuming the tortuosity factor equals one) the number of pores can be estımated. From the number of pores and their sizes the surface porosity is calculated to be $0.5 \%$, a very low value, as is also found for other UF membranes [19,20]

From thermoporometry and the gas adsorption-desorption technique it was found that PPO membranes possess a very sharp pore size distribution, with a characteristic mean pore

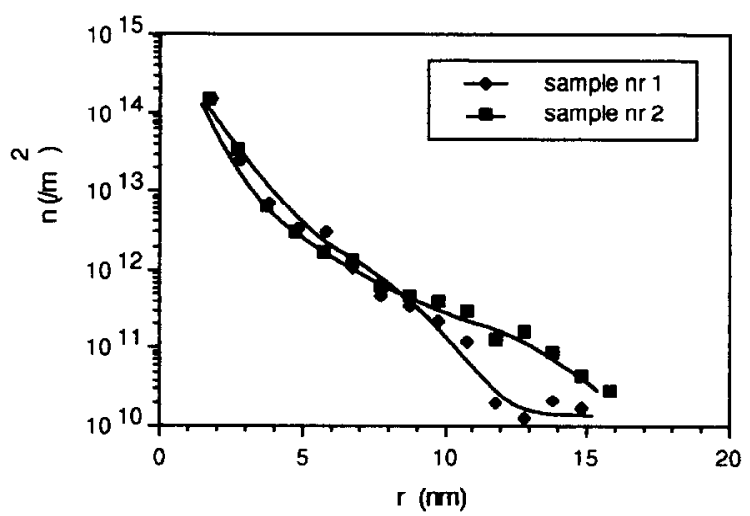

Fig 8 Pore size distribution using permporometry found for two samples of the same PPO membrane (lab-made), methanol was used as the adsorbate size of $2 \mathrm{~nm}$. This result is in strong contrast with the permporometry data mentioned above. Furthermore, the value of the total pore volume calculated from permporometry is 2000 times smaller than the value found by thermoporometry (about $1.5 \times 10^{-4} \mathrm{~cm}^{3} / \mathrm{cm}^{2}$ [21]) This can be explained by the fact that in the case of gas adsorption-desorption and thermoporometry the membrane is characterized as a whole (top- and sublayer) and small pores, which may be present in the sublayer, are measured too In the case of PPO membranes, the ratio between the porosity related to active pores (determined by permporometry) and the overall porosity (determined by thermoporometry) indicates that $9995 \%$ of the pores are present in the sublayer and consequently do not influence membrane performance. On the other hand, from permporometry it is suggested, that the pore volume of the larger active pores present in the skin, is so small that their presence cannot be detected by, e g. thermoporometry

\section{PSf membranes}

Pore size distributions of two PSf membranes are given in $F_{1 g} 9$ Methanol and ethanol, used as the condensable gases, showed

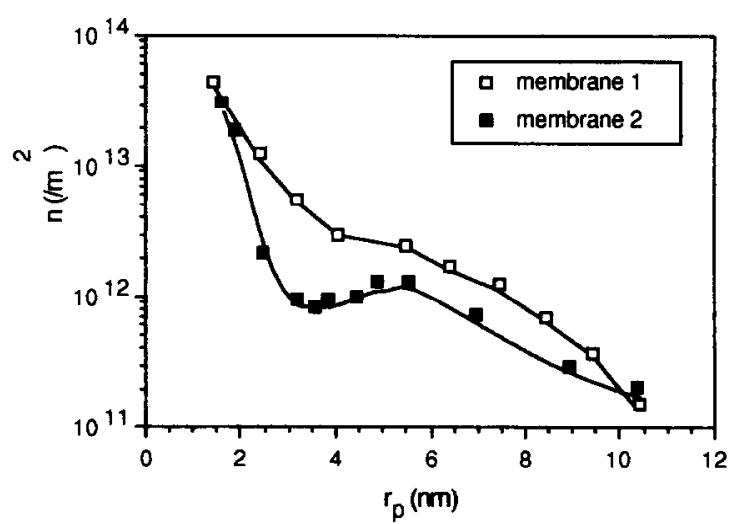

Fig 9 Pore size distribution using permporometry found for two lab-made PSf membranes (cast from the same polymer solution), ethanol was used as the adsorbate 
a similar t-layer thickness as in the case of PPO. Also for PSf membranes a broad pore size distribution is calculated (skin thickness $0.2 \mu \mathrm{m}$, determined with the gold sol method [18], $\tau=1$ ), with the largest pore size being approx. $10 \mathrm{~nm}$. Compared to PPO membranes, the number of the smaller pores ( $<5 \mathrm{~nm})$ is substantially lower. In Fig. 9 is shown, that despite the fact that the two membranes presented here were cast from the same polymer solution, their pore size distributions differ somewhat. One of the membranes appears to have a certain dip in the distribution at a pore size of about $3 \mathrm{~nm}$. This altogether indicates that, even for membranes made at nearly the same time and from the same polymer solution, differences in pore structure can be present. This sort of anomalies have also been shown by Nilsson [22].

The low surface porosity (calculated value $\sim 0.3 \%$ ) is probably the main reason that the pore size distribution of PSf membranes could not be determined by one of the methods already mentioned. This independence of pore volume, and its high resolution for open interconnected pores is another advantage of permporometry, in particular when UF membranes, which are known to have a low skin porosity, are evaluated.

\section{DDS GR61PP}

GR61PP membranes, manufactured by DDS, have a claimed cut-off of $20 \mathrm{kDa}$. The pore size distribution as determined with permporometry is remarkably sharp (Fig. 10). Most of the pores have sizes between 1.5 and $5 \mathrm{~nm}$, although a few pores with a size larger than 10 $\mathrm{nm}$ were found. Estimating the thickness of the top layer of the membranes, hence the length of the pores (with $\tau=1$ ), to be $0.2 \mu \mathrm{m}$, and using the number of pores calculated (Fig. 10), the surface porosity was calculated to be about $1 \%$.

The pore sizes (Fig. 10) found in this work

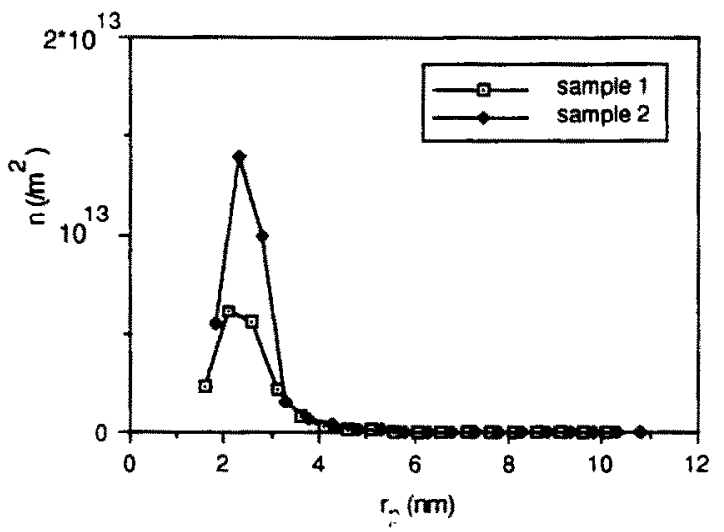

Fig. 10. Pore size distribution using permporometry found for two samples of the same DDS GR61PP membrane, ethanol was used as the adsorbate.

are somewhat lower than the values found by Hanemaaijer et al. [23] and Nilsson [22]. Hanemaaijer et al. used rejection measurements of low molecular weight sugar molecules [23], whereas Nilsson [22] determined the pore size distribution of the same type of membranes with the liquid-liquid displacement technique. Although their results are not comparable, both found that a considerable number of large pores were present. The 'characteristic pore size' of Hanemaaijer was about $10 \mathrm{~nm}$ (radius), the pore sizes of Nilsson ranged from $25 \mathrm{~nm}$ to 100 $\mathrm{nm}$. Nilsson also found that the porosity (number of pores) of membranes is inhomogeneous: the pore number on one part of the membrane differs significantly from another part. The latter is confirmed by permporometry measurements (Fig. 10), but pore sizes larger than 10 $\mathrm{nm}$ were not detected. The large differences in pore size might be due to the use of the different characterization techniques. The liquid-liquid displacement technique, used by Nilsson can cause swelling of the polymer, an effect that may induce larger pore sizes [3]. Such swelling effects might also play a role in permporometry measurements, but this was not found in the analysis of previous experiments using PPO and PSf membranes [19,21]. 


\section{Some comments on the pore size distribution determination by permporometry}

The distributions determıned for the different polymeric membranes are characterized by an asymmetric shape. For all the membranes, it appears that a relatively large number of small pores $(r<2 \mathrm{~nm})$ are present. The question arises whether this porosity really corresponds to such small pores or is an artefact induced by the characterization technıque itself.

One should realize that in the concept used here, the discrimination between t-layer desorption (or adsorption) and the capillary condensation is only an estimate. In case of the ceramic membranes the adsorption and desorption branch can be separated easily, as shown in Fig. 3. The lowest relative pressure where the adsorption and desorption process meet, is directly related to the point were capillary condensation and adsorption processes merge. For these membranes this transition is sharp because the pore size is well-defined. The question, however, is what happens when a wider pore size distribution and a relatively large number of small pores are present. In such a case the t-layer analysis proposed in this work neglects the presence of these small pores in favour of a larger t-layer thickness.

The range over which the Kelvin relation is valıd is not strictly defined, but it is generally agreed that for pores with radii smaller than 1.5 $\mathrm{nm}$, the equation is not valid. This can be rationalized because in these very small pores (about the size of a few molecules), the concept of a meniscus becomes meaningless [9]. In very small pores (micropores), the interaction between molecules and pore wall will be much higher than in mesopores. In such small pores adsorption already occurs at very low pressures and molecules present inside the pores can only be removed by using very low relative pressures $\left(p_{\mathrm{r}}=0\right.$ or in vacuum) This is not only true for the adsorbates used in this study, but also when such membranes are used in actual applications. This means that the transport of molecules in such pores is hindered very much. Together with the fact that in UF membranes the larger pores will mainly determine transport properties and hence the performance of the membrane, it can be concluded that even when small pores are present permporometry indeed gives relevant data on the structure of the membrane [21].

\section{Conclusions}

Permporometry is a method by which the active pore size distribution can be calculated accurately. Characteristic data found by permporometry, sometimes deviate substantially from the characteristics determined by other methods. These discrepancies can be fully understood from the pore structure itself.

The pore size distribution of ceramic $\gamma$-alumina membranes is very narrow, whereas the distributions of polymeric UF membranes are substantially broader. From the latter it can be concluded that the actual performance of polymeric membranes is governed by the larger pore sizes.

\section{List of symbols}

$p_{\mathrm{r}} \quad$ relative pressure (-)

$\theta \quad$ contact angle $\left({ }^{\circ}\right)$

$\gamma$ interfacial tension $\left(\mathrm{N} / \mathrm{m}^{2}\right)$

$r_{\mathbf{k}_{1}}$ (Kelvin) radius describing the curvature of the liquid-gas interface $(\mathrm{m})$

$v$ molar volume $\left(\mathrm{m}^{3} / \mathrm{mol}\right)$

$J_{\mathrm{k}}$ diffusive flux (mol/sec-Pa-m $\left.\mathrm{m}^{2}\right)$

$n$ number of capillaries per unit membrane area $\left(1 / \mathrm{m}^{2}\right)$

$r$ pore radius $(\mathrm{m})$

$\Delta p \quad$ partial pressure difference $(\mathrm{Pa})$

$l \quad$ thickness of the porous medium (m) 


\section{$M \quad$ molar mass of the gas $(\mathrm{g} / \mathrm{mol})$ \\ $\tau$ tortuosity (-)}

\section{References}

1 H Yasuda and J T Tsal, Gas permeability data used for the characterization of UF membranes, Appl Polym Scr , 18 (1974) 805

2 F W Altena, H A M Knoef, H Heskamp, D Bargeman and C A Smolders, Some comments on the applicability of the gas permeation methods to characterize porous membranes based on improved experimental accuracy and data handling, J Membrane Scı , 12 (1983) 313

3 H Bechhold, M Schlesinger and K Silbereisen, Porenweite von Ultrafiltern, Kolloid Z , 55 (1931) 172

4 S Munari, A Bottıno, P Morett1, G Panannell and I Becchi, Permporometric study on ultrafiltration membranes, J Membrane Sc1, 41 (1989) 61

5 Ch Eyraud, M Betemps and J F Quinson, Determ1nation des rayon de repartition de rayon des pores d'un ultrafiltre, Bull Soc Chım France, 9-10 (1984) 1-238

6 Ch Eyraud, Permporometry, A method to measure active pores, Paper presented at the Summer School on Membrane Science, Cadarache, 1984

7 A Mey-Marom and M Katz, Measurement of the active pore size distribution of microporous membranes A new approach, J Membrane Sc1, 27 (1986) 119

$8 \mathrm{M} \mathrm{Katz}$ and G Baruch, New insights into the structure of microporous membranes obtained using a new pore size evaluation method, Desalination, 58 (1986) 199

9 S J Gregg and K S W Sing, Adsorption, Surface Area and Porosity, 2nd edn , Academic Press, New York, NY, 1982

10 E A Mason and A P Malinouskas, Gas transport in Porous Media the Dusty Gas Model', Elsevier, Amsterdam, 1983
11 K K Keizer and A J Burggraaf, New ceramic membranes, in B Taylor (Ed ), Science of Ceramics, Vol 14, Institute of Ceramics, Shelton, UK, 1988, p 83

12 A Leenaars, Ceramic membranes, Thesis University of Twente, The Netherlands, 1984

13 M Katz, Measurement of the active pore size distribution of microporous membranes a new approach, Proc of the 3rd World Filtration Congress, 1982, p 508

14 B R Jones and W H Wade, in F M Fowkes (Ed), Hydrophobic Surfaces, Academic Press, New York, NY, 1969, p 206

$15 \mathrm{~J} B$ Perı, The reactıons of methanol over catalytic alumina, J Phys Chem, 70 (1966) 3168

16 A Hernandez, F Martinez-Villa, J A Ibanez, J I Arribas and A F Tejerine, An experımentally fitted and simple model for pores in nuclepore membranes, Sep Sc1 Technol , 21 (1981) 665

17 B D Michell and W M Deen, Effect of the concentration on the reflection coefficient of ngid macromolecules in track-etch membranes, J Colloid Interface $\mathrm{Sc}, 113$ (1986) 1

18 F P Cuperus, D Bargeman and C A Smolders, A new method to determine the skin thickness of anisotropic UF membranes using colloidal gold partıcles, J Collo1d Interface Sc1 , 135 (1990) 486

19 F P Cuperus, Characterization of UF membranes pore structure and top layer thickness, Thesis University of Twente, The Netherlands, 1990

20 A G Fane and C J D Fell, A review of fouling and fouling control in UF, Desalination, 62 (1987) 117

21 F P Cuperus and C A Smolders, Characterization of UF membranes membrane characterization and characterization techniques, Adv Collond Interface Sc1 , 34 (1991) 135

22 J L Nilsson, A study on membrane fouling, Thesis University of Lund, Sweden, 1989

23 J H Hanemaayer, T Robbertson, Th van den Boomgaard, C Olieman, P Both and D G Schmidt, Characterization of clean and fouled ultrafiltration membranes, Desalination, 68 (1988) 93 